\title{
Lymphoepithelioma-Like Carcinoma of Parapharyngeal Space-A Case Report with Review of Literature
}

\author{
Produl Hazarika ${ }^{1}$, Seema Elina Punnoose ${ }^{1}$, John Victor ${ }^{1}$, Sreekala ${ }^{2}$, Nirmali Dutta ${ }^{3}$ \\ ${ }^{1}$ Department of ENT, NMC Specialty Hospital, Abu Dhabi, UAE \\ ${ }^{2}$ Department of Pathology, NMC Specialty Hospital, Abu Dhabi, UAE \\ ${ }^{3}$ Department of Radiology, NMC Specialty Hospital, Abu Dhabi, UAE \\ Email: ${ }^{*}$ produl ent@rediffmail.com
}

Received 3 March 2015; accepted 20 March 2015; published 25 March 2015

Copyright (C) 2015 by authors and Scientific Research Publishing Inc.

This work is licensed under the Creative Commons Attribution International License (CC BY).

http://creativecommons.org/licenses/by/4.0/

(c) (i) Open Access

\begin{abstract}
Extra-nasopharyngeal lymphoepithelioma-like carcinomas (LELC) are uncommon epithelial tumors. A few isolated case reports and series are available in literature involving the larynx, pharynx, salivary gland, lung etc., but involvement in the parapharyngeal space has not yet been reported. We aim to highlight one such case that has a clinical and radiological characteristic of a benign lesion whilst the histopathology reveals an infiltrating neoplasm. The typical clinical aggressiveness of a classical LELC of extra-nasopharyngeal lesion as described in literature is not present in our case. Also seen is an uncommon finding of abnormal branching of left external carotid artery. There is no standard treatment protocol for such a tumor; however, wide excision of this tumor in the parapharyngeal space via trans-cervical, trans-mandibular, trans-palatal approach has shown good and satisfactory tumor control of the primary site so far.
\end{abstract}

\section{Keywords}

Lymphoepithelioma-Like Carcinoma (LELC), Parapharyngeal Space, Trans-Cervical, Trans-Mandibular, Cells in Cords, Lymphoplasmacytoid

\section{Introduction}

Parapharyngeal space tumors are rare and comprise only $0.5 \%$ of all head and neck tumors and a vast majority of these are benign in nature [1]. Even more rare is a primary malignant tumor of parapharyngeal space. Our se-

\footnotetext{
${ }^{*}$ Corresponding author.
}

How to cite this paper: Hazarika, P., Punnoose, S.E., Victor, J., Sreekala and Dutta, N. (2015) Lymphoepithelioma-Like Carcinoma of Parapharyngeal Space-A Case Report with Review of Literature. International Journal of Otolaryngology and Head \& Neck Surgery, 4, 163-168. http://dx.doi.org/10.4236/ijohns.2015.42028 
nior author [2] has published a series of 41 cases of parapharyngeal tumor where he found only 5 cases with malignant pathology. Herein reported is a rare case of lympho-epithelioma-like carcinoma (LELC) primarily arising from the parapharyngeal space having no clinical or radiological evidence of involvement of the nasopharynx. This neoplasm seems to have behaved in a fashion remniscent of nasopharyngeal carcinoma. However, predominant association of Ebstein Barr Virus in nasopharyngeal lymphoepithelioma is not seen commonly in LELC of extranasopharyngeal origin. A review of available English literature using search engines Medscape and Pubmed failed to show any previous report of LELC in parapharyngeal space. This case study of lymphoepithelioma-like carcinoma in the parapharyngeal space is peculiar and unusual. The preoperative diagnosis of this neoplasm was difficult and final definitive diagnosis was established only after immunohistochemistry and electron microscope studies. In the present case, the diagnosis is supported by histopathological studies and immunohistochemistry. There was no associated tumor involvement of nasopharynx or lung on clinical and radiological examination. The idea of presenting this case is to highlight its rare occurrence of this tumor in the parapharyngeal space, its diagnostic as well as management dilemmas along with the abnormal branching of external carotid artery. This could very well be the first reported case of primary lymphoepithelioma-like carcinoma found in the parapharyngeal space to the best of our knowledge.

\section{Case Report}

A 30 years old Indian male patient attended the ENT Clinic of NMC Specialty Hospital, Abu Dhabi, UAE on 7th October 2013 for his complaints of breathing difficulty, huskiness of voice and snoring during sleep of 6 months duration. There was no difficulty in swallowing but his initial symptoms of breathing difficulty aggravated in the last one month. Clinical examination revealed a globular, smooth and congested mass pushing the left side of soft palate and uvula downwards and medially causing narrowing of the oropharyngeal inlet. A rigid videolaryngoscopy showed the lower margin of the mass going beyond the left pharyngoepiglottic fold. The supraglottic larynx was pushed to the right side due to mass effect (Figure 1). A CT scan Neck with contrast was done on 10th October 2013 which revealed a well-defined elongated spindle shaped enhancing soft tissue mass in the left parapharyngeal space measuring $7.5 \mathrm{~cm}$ cranio-caudally, $3 \mathrm{~cm}$ antero-posteriorly and $4.5 \mathrm{~cm}$ transversely. The mass was extending from jugular foramen superiorly to the level of hyoid bone inferiorly. Medially, this mass was causing medial displacement of the visceral space with indentation on the left lateral wall of nasopharynx, oropharynx and hypopharynx (Figure 2). His hematological and biochemical investigations were within normal limits. We decided on an excision biopsy instead of FNAC and patient was posted for surgery on

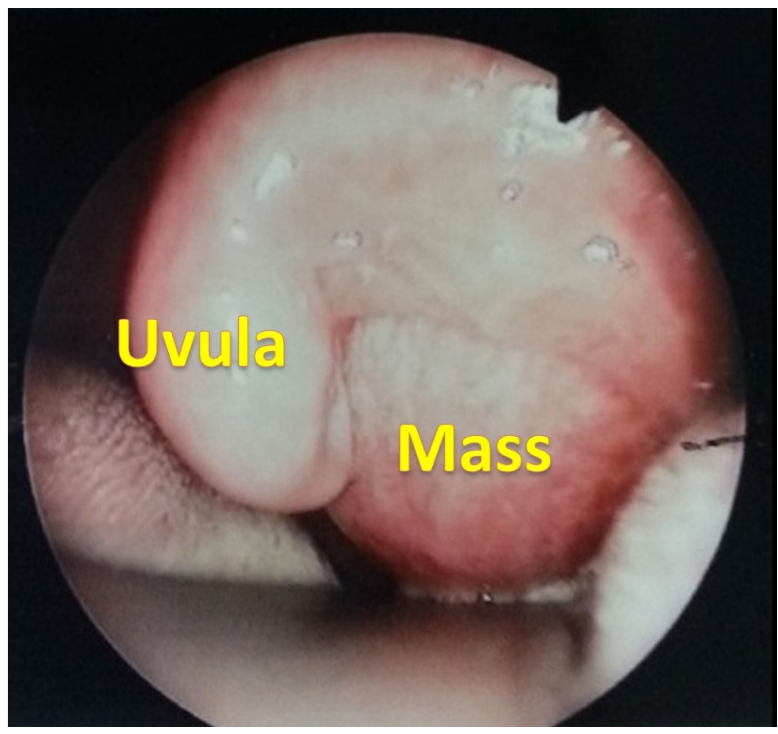

(a)

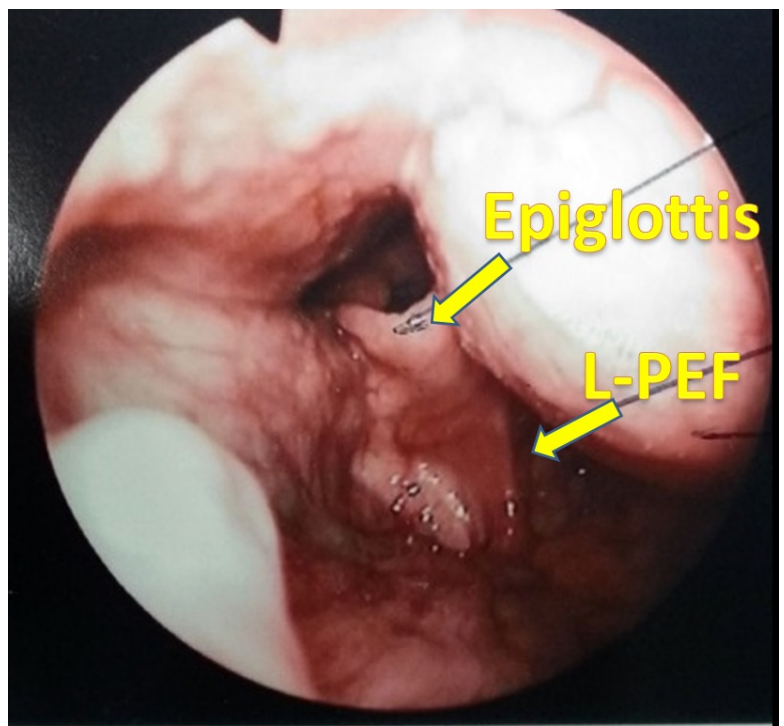

(b)

Figure 1. (a) Rigid videolaryngoscopy: the lower margin of the mass going beyond the left pharyngoepiglottic fold with mass effect pushing the supraglottic larynx to right side; (b) Globular, smooth and congested mass pushing the left side of soft palate and uvula downwards and medially with narrowing of the oropharyngeal inlet. 


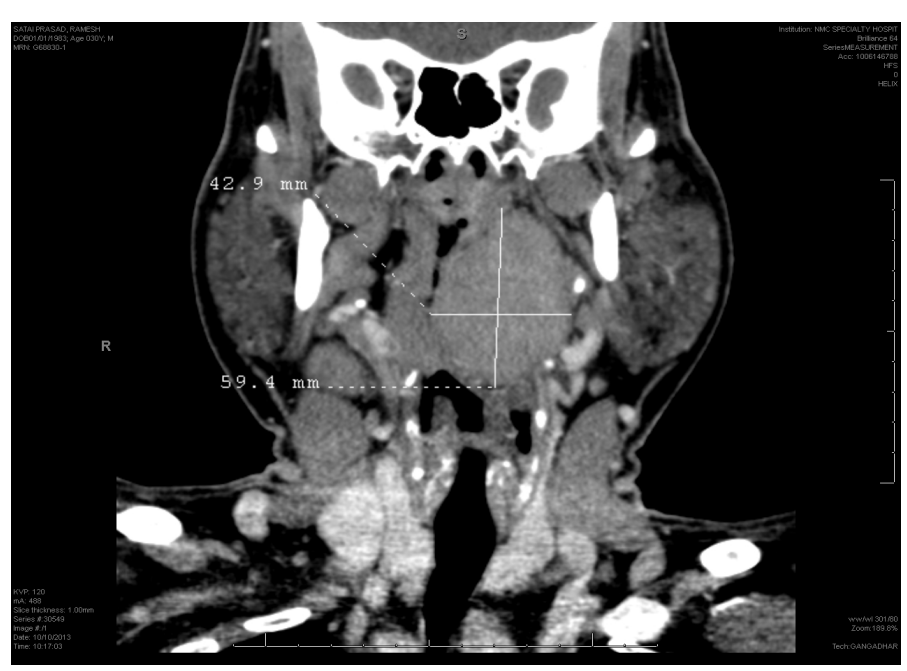

Figure 2. CT scan neck with contrast coronal cut: Well-defined elongated spindle shaped enhancing soft tissue mass in the left parapharyngeal space.

11th November 2013. Trans-cervical, trans-mandibular approach with lateral paramedian mandibulotomy was adopted for resection of the tumor. Intraoperatively, we encountered abnormal branching of left superior thyroid and left lingual artery from the left carotid bulb (Figure 3(a) and Figure 3(b)). The tumor was firmly adherent superiorly to the base of the skull and had to be removed in piecemeal at the superior pole in the area of attachment only and rest of the tumor was removed as a single well encapsulated tumor (Figure 4). A few level I and II cervical lymph glands were found on the left side which was removed and sent for histopathology along with the excised main mass. Histopathology showed a well encapsulated tissue with an infiltrating neoplasm of cells arranged in cords, nests and sheets in a dense lymphoplasmacytoid background. The cells had moderate to abundant eosinophilic cytoplasm with pleomorphic hyperchromatic nuclei having nucleoli. Dyskeratotic cells were also noted. The mitoses were frequent with apoptotic debris. The excised 5 cervical lymph glands were reactive in nature only. The histological diagnosis turned out to be lymphoepithelioma-like carcinoma of undifferentiated type. Due to the rarity of the lesion, the histopathological slide was further evaluated in the pathology department of Manipal University, India where the diagnosis was reconfirmed. Immunohistochemical analysis showed that the cells were positive for CK 5/6, PanCK and p63 and negative for CD3, CD20, S100 and CEA (Figure 5(a) and Figure 5(b)).

\section{Discussion}

Parapharyngeal tumours are mostly benign. Some published reports have commonly reported neurogenic tumours as being frequently seen. While various other authors in their series have reported salivary gland tumors as the most commonly encountered tumors in the parapharyngeal space. Malignant tumors are very rarely reported [2]-[4].

Lymphoepithelioma-like carcinoma (LELC) is a different histopathological entity having microscopic resemblance to nasopharyngeal lymphoepithelioma. This entity is a histological variant of malignant tumor arising from uncontrolled mitosis of the transformed cells originating in the epithelial tissue. However, LELC is believed to arise outside the nasopharynx yet resembling histologically with nasopharyngeal lymphoepithelioma [5].

LELC was first described in 1921 as a variant of squamous cell carcinoma. LELC grows either in nests or as a single infiltrating cell with cells round or oval vesicular nuclei, prominent nucleoli, indistinct cytoplasmic borders and numerous mitoses [6]. The cells are undifferentiated and non-keratinizing. It stains positive for epithelial marker such as cytokeratin and epithelial membrane antigen. Usually an inflammatory infiltrate rich in mature lymphocytes (predominantly $\mathrm{CD}^{+}$and $\mathrm{T}$ cells) and occasionally eosinophils are seen, particularly obscuring the neoplastic epithelial component [7]. Conventionally, LELC is regarded as high grade based on poor histological differentiation. 


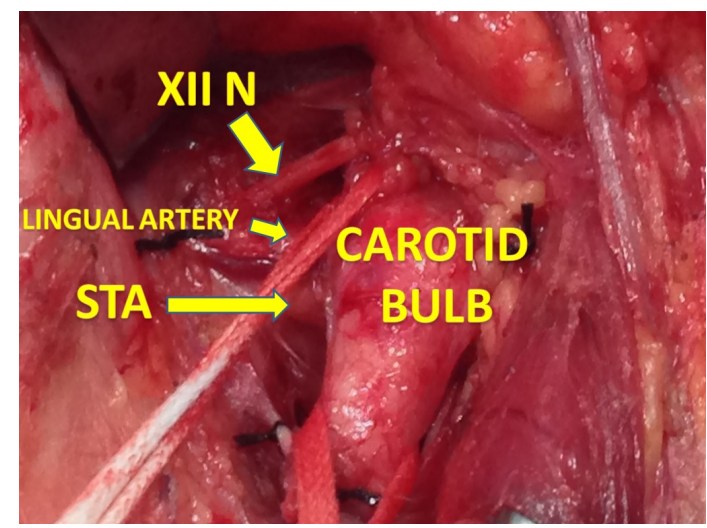

(a)

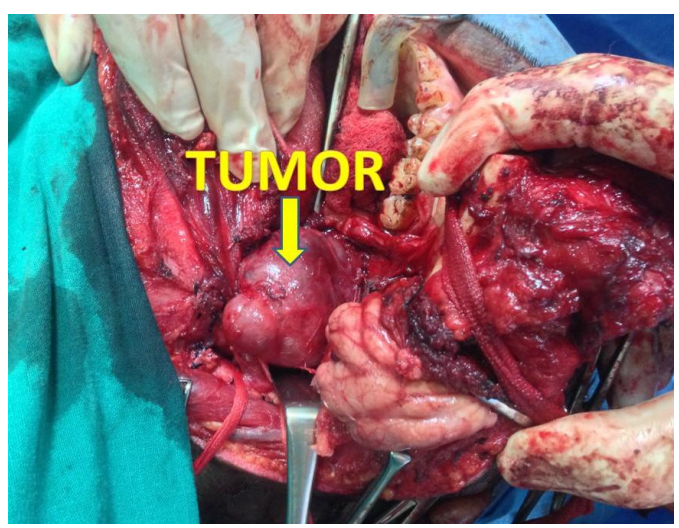

(b)

Figure 3. (a) An abnormal branching of left superior thyroid and left lingual artery from the left carotid bulb; (b) Intraoperative view of tumour exposed via transcervical transmandibular approach; the extent of the mass seen in the base of skull.

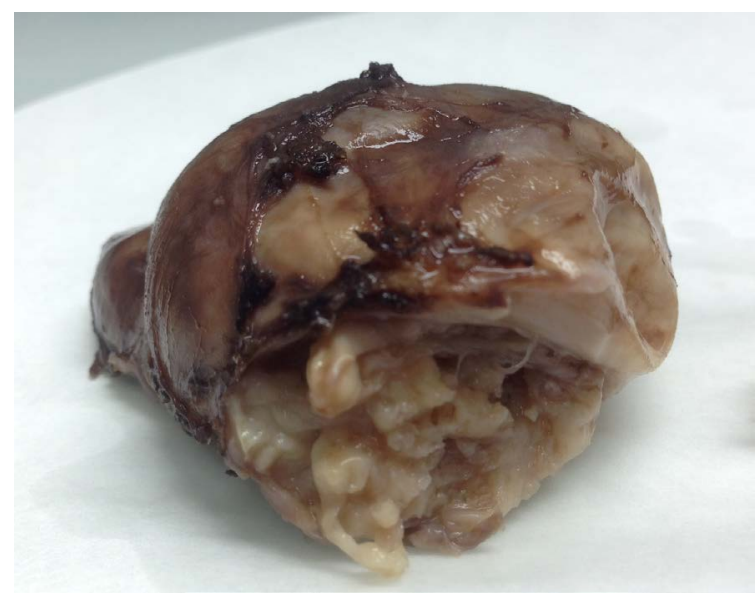

Figure 4. Post operative specimen showing single well encapsulated tumour removed from left parapharyngeal space.

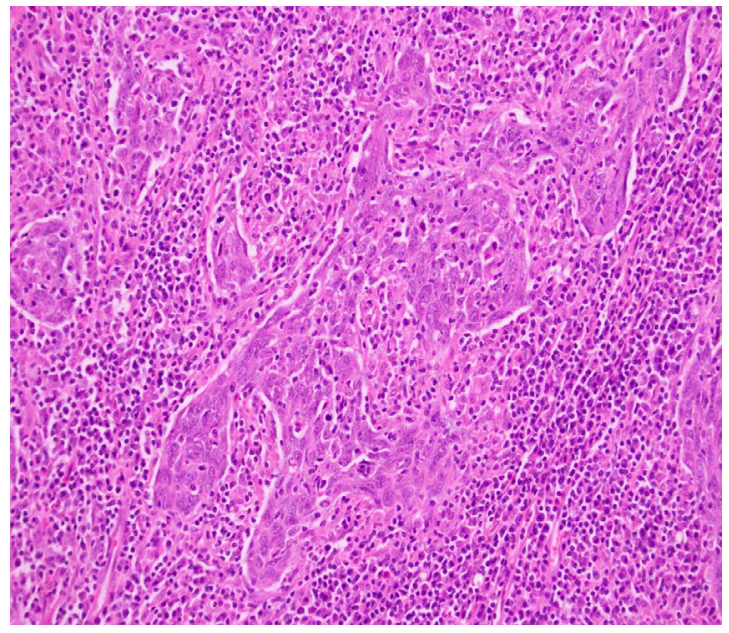

(a)

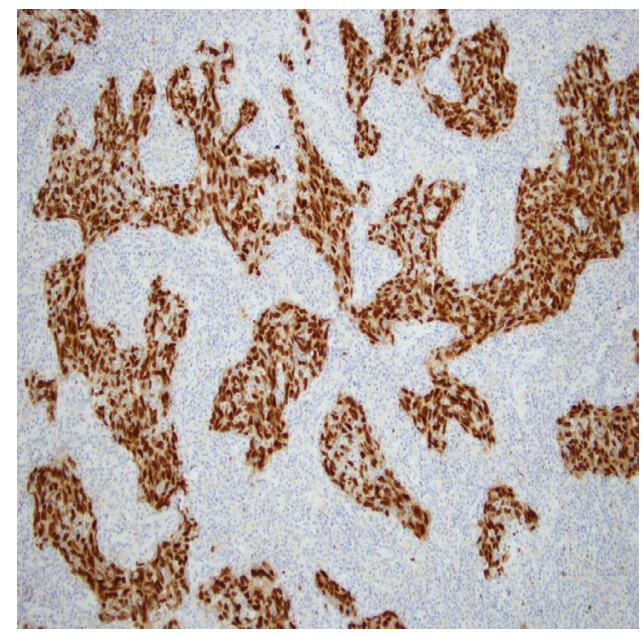

(b)

Figure 5. H \& E 200×; LEC; islands and nests of tumor cells in a lymphoid background p63 positive in tumor cells. 
LELC has a strong etiopathological association with EBV, mostly in South Eastern Asian population [8]. However, the connection between LELC and EBV is variable and may not always have any ethnic relevance. There have been reports of LELC arising from the pharynx or foregut derivatives in Asian population who are more prone for EBV than Caucasians [3]. The associations of EBV are more site specific and seen more in tumors arising from lung, stomach, thymus and salivary gland [9]. Circulating serum EBV DNA could be used as a tumor marker in the clinical management of LELCs. It is reported that patients with a pre-therapy serum EBV DNA more than 10,000 copies/ml had significantly worse survival rate [10]. Elevated levels 6 to 8 weeks after therapy were strongly associated with both progression free and overall survival [10] [11]. In our present case, circulating serum of EBV DNA was not done as our preoperative diagnosis was in favor of benign neurogenic tumor and hence was never thought of. In-situ hybridization (RNA-ISH) and polymerase chain reaction (PCR) for detection of Epstein Barr Virus genome in both epithelial and lymphoid population would have been more informative. Immunohistochemical studies done in our case shows cells were positive for tumor markers CK5/6, Pan CK and P63 and negative for CD3, CD20, S100 and CEA. These parameters are in favor of LELC.

Ma, Lin, Wang, et al. (2014) [12] reported sixty nine cases of lymphoepithelioma-like carcinoma from salivary gland but none of these are from the parapharyngeal space. CT scan neck and extensive sampling in histopathological studies in our case, failed to show any evidence of salivary gland tissue excluding the possibility of its origin from salivary gland. Extensive sampling was done from the post operative specimen after inking the surface. The lesion was well encapsulated and completely excised as shown in Figure 4.

Clinically, the most common symptom of a parapharyngeal tumour is a neck mass. Other symptoms include swallowing problems, feeling of obstruction and pain in the throat, unilateral tinnitus, trismus, dysarthia, glossopharyngeal neuralgia and cranial nerve palsies. Pain, trismus and cranial nerve palsies are often suggestive of malignancy. Our patient had initial symptoms of lump in the throat, huskiness of voice, mild breathing difficulty on exertion. No visible neck mass or gland was found. This could be because the tumor was arising high in the parapharyngeal space towards the skull base. However, the lump was seen on rigid laryngoscopy as a swelling in the left lateral pharyngeal wall pushing the supraglottic larynx towards the right side. The initial clinical impression in this case was favoring more towards benign tumors like schwanomma. LELC was the post operative histopathological diagnosis.

FNAC, MRI CT Scan Neck with or without contrast and Angiography are the common investigative tools used for parapharyngeal masses. In the present case, the classic CT angiographic findings pointed towards a benign tumor. Since the tumor was deep seated in the parapharyngeal space with a radiological evidence of benign character of tumor we decided excision biopsy as our ideal option rather than FNAC because of its high level of specificity.

Management of such tumors is difficult as there is no validated therapeutic management that exists for LELC. Surgical excision [6] has been reported as the best option in localized disease occasionally followed by radiotherapy [9]. Chemotherapy is not a conventional option. Taxanes, platinum derivatives and 5-fluorouracil combined with folinic acid are active drugs [13]. Surgical approaches for the excision of parapharyngeal tumors are numerous but trans-cervical approach is more popular because of its versatility. However, the surgical dilemma involved is in the selection of the appropriate approach while avoiding injury to the great vessels with preservation of functions of lower cranial nerves, with or without mandibulotomy and reduction of traction trauma to the soft tissues intraoperatively. In our present case, we found an unusual branching of left external carotid artery where the superior thyroid and lingual artery were branching from the left carotid bulb instead of the left external carotid artery. This kind of abnormal branching is rare but has been reported earlier [14]. So, meticulous dissection is also imperative to look out for any structural abnormality of vessels and nerves thus avoiding inadvertent injury.

LELC has generally been considered as highly radiosensitive and hence radiotherapy is to be considered especially for residual tumors, high grade tumors or tumors with only loco-regional metastasis. Prognosis depends on the stage and grading of the tumor. Our present case was treated with primary surgery. Radiotherapy will be a considered optiion if recurrence of the tumor is found in the follow up period. A repeat CT scan Neck done on 25th February 2014 showed no recurrence of the primary tumor in the parapharyngeal space and during his last visit on 3rd January 2015 to our outpatient department patient was asymptomatic and doing well. He is currently under our close watch and follow up.

\section{Conclusion}

Lymphoepithelioma like carcinoma has a close histopathological similarity with nasopharyngeal lymphoepithe- 
lioma and can arise primarily from extra nasopharyngeal sites like salivary gland, oropharynx, larynx, gastric mucosa, lung etc. However, LELC arising primarily from parapharyngeal space has not been reported in English literature. One such case is reported here because of its rarity and dilemma in management. Such diagnosis often requires immunohistopathology and electron microscopy for confirmation.

\section{Acknowledgements}

We, the authors acknowledge the immense help and support that we received from our NMC group medical director Dr. B. R. Shetty and Medical Director of NMC Specialty Hospital, Abu Dhabi Dr. C. R. Shetty without whom none of this would have been possible.

\section{References}

[1] Panda, N., Gosh, S., Jain, A. and Vadhishta, R. (2004) Unusual Malignant Tumours of the Parapharyngeal Space-A Diagnostic Dilemma. The Internet Journal of Otorhinolaryngology, 4, 1.

[2] Hazarika, P., Dipak, R.N., Parul, P. and Kailesh, P. (2004) Surgical Access to Parapharyngeal Space Tumors: The Manipal Experience. Medical Journal of Malaysia, 59, 323-329.

[3] Carru, R.L., Myers, E.N. and Johnson, J.T. (1990) Management of Tumors Arising in Parapharyngeal Space. Laryngoscope, 100, 583-489.

[4] Pensak, M.L., Gluckman, J.L. and Shumrick, K.A. (1994) Parapharyngeal Space Tumors: An Algorithm for Evaluation and Management. Laryngoscope, 104, 1170-1173. http://dx.doi.org/10.1288/00005537-199409000-00022

[5] Aurilio, G., Ricci, V., Devita, F., Fasano, M., Fazio, N., Orditura, M., Funicelli, L., De Lusa, G., Lasevoli, D., Lovino, F., Ciardiello, F., Conzo, G., Nole, F. and Lamendolla, M.G. (2010) A Possible Connective Tissue Primary Lymphoepithelioma Like Carcinoma. E-Cancer Medical Science, 4, 197.

[6] Bildirici, K., Ak, G., Peker, B., Metintas, M., Alatas, F., Erginel, S. and Ucgun, I. (2005) Primary Lymphoepithelioma of Lung. Tuberk Toraks, 53, 69-73.

[7] Kobayashi, M., Ito, M., Sano, K., Honda, T. and Nakayama, J. (2004) Pulmonary Lymphoepithelioma-Like Carcinoma: Predominant Infiltration of Tumor-Associated Cytotoxic T Lymphocytes Might Represent the Enhanced Tumor Immunity. Internal Medicine, 43, 323-326. http://dx.doi.org/10.2169/internalmedicine.43.323

[8] Lezzone, J.C., Gaffey, M.J. and Weiss, L.M. (1995) The Role of Epstein-Barr-Virus in Lymphoepithelioma Like Carcinoma. American Journal of Pathology, 103, 308-315.

[9] Nagan, R.K., Yip, T.T., Cheng, W.W., Chan, J.K., Cho, W.C., Ma, V.W., Wan, K.K., Au, J.S. and Law, C.K. (2004) Clinical Role of Circulating Epstein Barr Virus DNA as a Tumor Marker in Lymphoepitheloma Like Carcinoma of the Lung. Annals of the New York Academy of Sciences, 1022, 263-270. http://dx.doi.org/10.1196/annals.1318.041

[10] Griffin, B.E. and Xue, S.A. (1998) Epstein Barr Virus Infections and Their Association with Human Malignancy: Some Key Questions. Annals of Medicine, 30, 294-259. http://dx.doi.org/10.3109/07853899809005852

[11] Sun, X.N., Xu, J., Yang, Q.C., Hu, J.B. and Wang, Q. (2006) Lymphoepithelioma Like Carcinoma of the Salivary Gland: A Case Report. Chinese Medical Journal (English Edition), 119, 1315-1317.

[12] Ma, H., Lin, Y., Wang, L., Rao, H., Xu, G., He, Y. and Liang, Y. (2014) Primary Lymphoepithelioma-Like Carcinoma of Salivary Gland: Sixty Nine Cases with Long Term Follow-Up. Head \& Neck, 36, 1305-1312.

[13] Chan, A.T., Teo, P.M., Lam, K.C., Chan, W.Y., Chow, T.H., Yim, A.P., Mok, T.S., Kwan, W.H., Leung, T.W. and Johnson, P.J. (1998) Multimodality Treatment of Primary Epithelioma Like Carcinoma of the Lung. Cancer, 83, 925929. http://dx.doi.org/10.1002/(SICI)1097-0142(19980901)83:5<925::AID-CNCR18>3.0.CO;2-X

[14] Gluncic, V., Pentanjek, Z., Marusic, A. and Gluncic, I. (2001) High Bifurcation of Common Carotid Artery, Anomalous Origin of Ascending Pharyngeal Artery and Anomalous Branching Pattern of External Carotid Artery. Surgical and Radiologic Anatomy, 23, 123-125. http://dx.doi.org/10.1007/s00276-001-0123-x 